\title{
Advanced image guidance for prostatic artery embolization - a multicenter technical note
}

\author{
Francisco Cesar Carnevale ${ }^{1 *}$ (D) Timothy McClure ${ }^{2}$, Farah Cadour ${ }^{3}$, Vincent Vidal ${ }^{3}$, André Moreira de Assis ${ }^{1}$, \\ Airton Mota Moreira', Arthur Diego Dias Rocha', Aya Rebet ${ }^{4}$ and Charles Nutting ${ }^{5}$
}

\begin{abstract}
Background: Prostatic artery embolization (PAE) is associated with patients' quality of life improvements and limited side effects compared to surgery. However, this procedure remains technically challenging due to complex vasculature, anatomical variations and small arteries, inducing long procedure times and high radiation exposure levels both to patients and medical staff. Moreover, the risk of non-target embolization can lead to relevant complications. In this context, advanced imaging can constitute a solid ally to address these challenges and deliver good clinical outcomes at acceptable radiation levels.

Main text: This technical note aims to share the consolidated experience of four institutions detailing their optimized workflow using advanced image guidance, discussing variants, and sharing their best practices to reach a consensus standardized imaging workflow for PAE procedure, as well as pre and post-operative imaging.

Conclusions: This technical note puts forth a consensus optimized imaging workflow and best practices, with the hope of helping drive adoption of the procedure, deliver good clinical outcomes, and minimize radiation dose levels and contrast media injections while making PAE procedures shorter and safer.
\end{abstract}

Keywords: Prostatic artery embolization, Benign prostatic hyperplasia, Lower urinary tract symptoms, Arterial vascular anatomy, Cone-beam computed tomography

\section{Background}

With transurethral resection of the prostate (TURP) being associated with higher risks of major adverse events such as bleeding, urinary incontinence, retrograde ejaculation and impotence, prostatic artery embolization (PAE) has become an important alternative for patients with lower urinary tract symptoms (LUTS) due to benign prostatic hyperplasia (BPH) who have failed pharmacotherapy (Rassweiler et al. 2006; Carnevale et al. 2013; Gao et al. 2014; Carnevale et al. 2016; Lecumberri et al. 2018; Ray et al. 2018; Hacking et al. 2019; Carnevale et al. 2020). Its benefits for patients and cost

\footnotetext{
* Correspondence: francisco.carnevale@criep.com.br

${ }^{1}$ Department of Radiology, University of Sao Paulo Medical School, Av. Dr.

Enéas Carvalho de Aguiar, 255, São Paulo, SP 05403-000, Brazil

Full list of author information is available at the end of the article
}

effectiveness have been demonstrated (Bagla et al. 2017; Brown et al. 2019).

One of the major technical challenges in performing PAE is the identification of and navigation through the pelvic and prostatic vascular anatomy, which presents significant variability among individuals and between pelvic sides of the same patient (de Assis et al. 2015; Carnevale et al. 2017; Bilhim et al. 2011a). In particular, prostatic arteries have highly variable origins and are frequently small, tortuous and stenotic, especially in the elderly population (de Assis et al. 2015). Although rare (Carnevale et al. 2020), non-target embolization can lead to significant complications such as rectal and bladder ischemia or penile ulcers. This challenging procedure therefore requires both excellent vascular anatomy understanding with microcatheterization skills and high 
precision in selecting the injection points to deliver the embolic material without reflux nor through arterialarterial shunts (de Assis et al. 2015).

Although the use of cone-beam computed tomography $(\mathrm{CBCT})$ has recently been encouraged to better understand the pelvic vascular anatomy (Bagla et al. 2013; Wang et al. 2017; Rocha et al. 2020; Cadour et al. 2020; Bagla and Sterling 2014; Schnapauff et al. 2020), PAE remains technically challenging, typically resulting in long procedure and fluoroscopy times, and high radiation dose due to the need for multiple oblique digital subtraction angiographies (DSA) and magnified views, with significant variability between centers (median DAP ranging from 33.2 to $863.4 \mathrm{~Gy} \cdot \mathrm{cm} 2$ between individual studies) (Laborda et al. 2015; Garzon et al. 2016; Andrade et al. 2017; Maclean et al. 2017; Tanaka et al. 2017; Zumstein et al. 2020). In this context, a refined technique is crucial for technical and clinical success. If the embolization technique has been well described (Cornelis et al. 2020; Carnevale et al. 2014), little has been said about image guidance available to the Interventional Radiologist.

This technical note aims to share the consolidated experience of physicians from four institutions performing a total of 300 PAEs per year, on average. A consensus imaging workflow, optimized for PAE, is described, some variants discussed, and best practices using advanced image guidance shared, in hopes of providing the Interventional Radiology community with a resource to deliver good PAE technical and clinical outcomes at acceptable radiation dose levels.

This retrospective analysis was approved by local institutional review boards and exempted from patient informed consent.

\section{Consensus imaging workflow Methods for reaching consensus}

Six PAE users from four institutions, with 4 to 13 years of experience in PAE, gathered to discuss their specific techniques and propose a consensus imaging workflow. Reviews were organized for each user to present their imaging workflow and technique, allowing to highlight commonalities and differences and to share best practices, which were then prospectively tested by the other authors to feed the following discussions, resulting in an optimized imaging workflow and technique adopted by all centers (Fig. 1). This workflow was established with the objective of better visualizing the anatomy, better planning, guiding and assessing the procedure, while minimizing radiation exposure to patients and clinical staff.

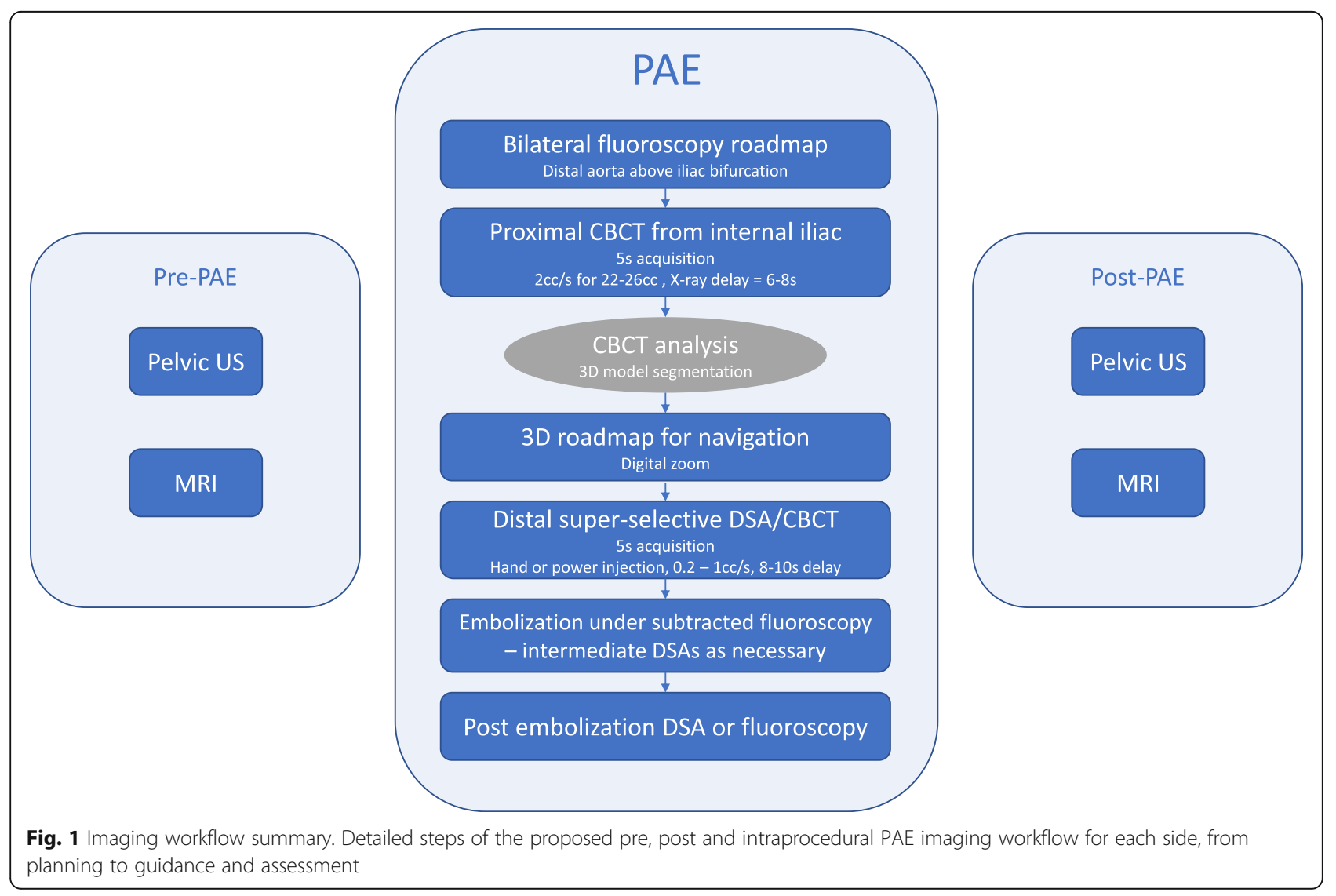




\section{Pre-operative imaging}

Understanding the specific patient pelvic anatomy is an important step of the procedure planning phase (de Assis et al. 2015). Pre-procedural computed tomography (CT) and magnetic resonance (MR) angiography can be useful to understand the main prostatic artery supply (Bilhim et al. 2011b; Kim et al. 2018). However, the nonselective injection and CT's and MR's limited spatial resolution make the identification of small prostatic arteries challenging (Maclean et al. 2018), with intraoperative $\mathrm{CBCT}$ shown to allow for better prostatic artery identification with improved signal-to-noise and contrast-to-noise ratio and with less radiation dose compared to conventional CT (Desai et al. 2018). In all centers experience, CT and MR's spatial resolution was too limited for detailed anatomical analysis (e.g. when trying to localize prostatic artery origins and distal connections, anatomy pathway, and anastomoses with neighboring structures). With superior contrast resolution, preoperative MR is still recommended to confirm $\mathrm{BPH}$, rule out prostate cancer, estimate zonal and total prostatic volumes and plan for PAE treatment, especially for smaller prostates (Guneyli et al. 2016; de Assis et al. 2017). Reasons to use pre-PAE CTA would include understanding of the pelvic and main prostatic vascular anatomy, selection of arterial access (femoral or radial) and selection of catheters to be used during the procedure. However, intra-procedural $\mathrm{CBCT}$ is the best imaging tool to understand the prostatic arteries, intraprostatic anastomosis and the prostate zone that each prostatic branch is feeding. Pre-operative CT is not recommended to minimize overall patient radiation exposure and contrast. Pelvic US is recommended to measure the postvoid residual volume.

\section{Intraprocedural imaging - general guidelines}

During PAE procedure, DSA offers excellent spatial resolution from selective injection points; however, the projective nature of DSA images can be misleading when analyzing small and tortuous prostatic arteries, prompting the needs for multiple projections, which typically results in increased levels of radiation exposure, contrast medium and procedure time. CBCT has been increasingly adopted and recommended during IR procedures, allowing a precise assessment of complex vascular anatomy in $3 \mathrm{D}$ with a single injection of contrast medium in a selectively targeted artery. Its benefits include high spatial resolution combined with an intra-arterial injection of a smaller volume of contrast compared to CT. Its value during PAE compared to preoperative $\mathrm{CT}$ and to DSA has already been demonstrated (Bagla et al. 2013; Wang et al. 2017; Rocha et al. 2020; Cadour et al. 2020; Bagla and Sterling 2014; Schnapauff et al. 2020; Desai et al. 2018). Compared to DSA, CBCT provided information that impacted treatment in $46 \%$ of PAE patients by allowing identification of potential sites of nontarget embolization (Bagla et al. 2013). Recent studies concluded that the number of prostatic arteries origins and anastomoses that could be identified were significantly higher with CBCT than with DSA (Wang et al. 2017), and that CBCT provided essential information that was not available with DSA in 46\% (Rocha et al. 2020) to $60.8 \%$ (Wang et al. 2017) of patients. Finally, advanced imaging techniques such as 3D road-mapping should be utilized as they can contribute to both performing safer and more efficient procedures, and reducing $\mathrm{x}$-ray dose and procedure time (Hertault et al. 2018; Schott et al. 2019).

\section{Intraprocedural imaging detailed workflow}

To provide a complete overview of the pelvic anatomy, an initial bilateral non-selective fluoroscopic roadmap is recommended from the distal aorta just above the aortic common iliac bifurcation. This roadmap is helpful in understanding internal iliac arteries origin as well as in providing the length of the common and internal iliac arteries to support catheter selection (Laborda et al. 2015). In this group's experience, hand-injected fluoroscopic roadmap of $50 \%$ diluted contrast provides sufficient image quality at this stage, and decreases radiation exposure compared to DSA.

PAE usually consists of a bilateral successive embolization, often starting from the left side because of the designed use of the Robert's uterine and the Carnevale's prostate catheters when performed through the femoral approach. For each side, a first proximal $5 \mathrm{~s}$ $\mathrm{CBCT}$ is acquired with the 5 French catheter in the internal iliac artery, above the bifurcation of the anterior and posterior branches (Carnevale et al. 2014). The following injection parameters are typically used: 22 to 26 cc of pure contrast, injected at $2 \mathrm{cc} / \mathrm{s}$, with an X-ray delay of 6 to $8 \mathrm{~s}$. This injection protocol ensures a good filling of the arteries during the entire spin, thus allowing an adequate visualization of both the arterial anatomy and the prostate parenchymal blush in a single CBCT (Fig. 2).

With the 180 degrees rotational DSA-like image provided by the CBCT spin, and with recent studies showing CBCT's superiority to DSA for PAE planning (Wang et al. 2017; Rocha et al. 2020), it is recommended to skip the typically acquired ipsilateral oblique DSA from the internal iliac artery (Carnevale and Antunes 2013) to limit patient radiation exposure, contrast medium use and reducing the procedure time. Another variant to further reduce radiation dose consists of acquiring the proximal CBCT bilaterally from the distal aorta, with 60 cc of contrast injected at $6 \mathrm{cc} / \mathrm{s}$ with an X-ray delay of 6 s. In the group's experience, this CBCT does not provide 


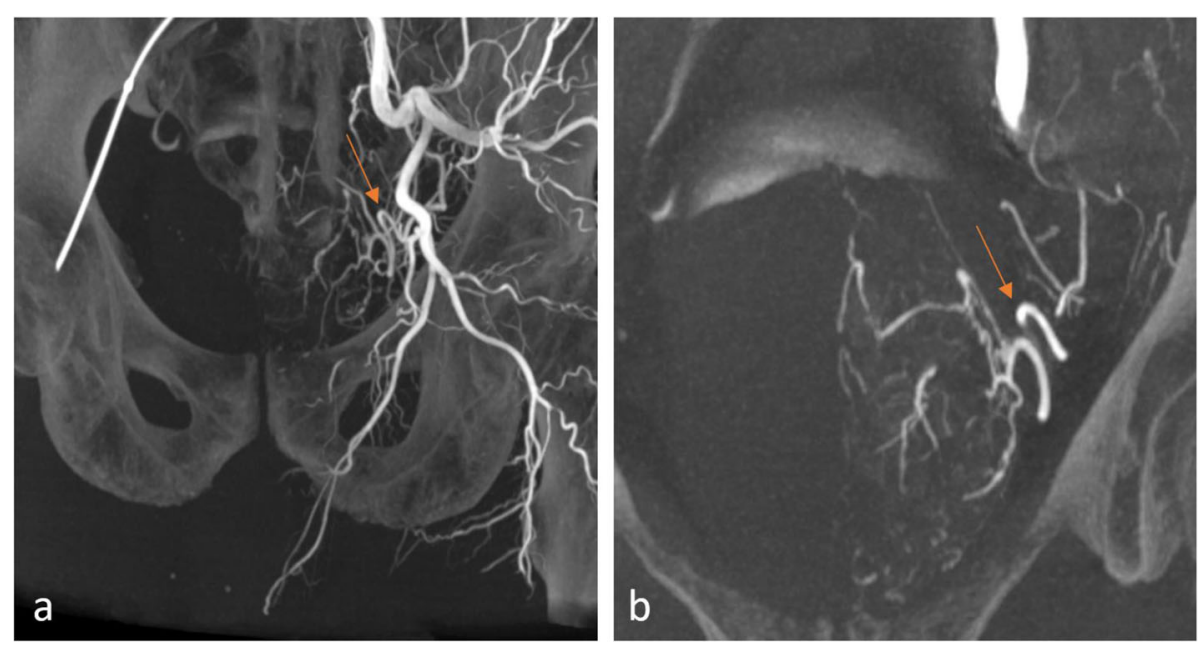

Fig. 2 Proximal CBCT. Five seconds CBCT acquisition, injection from left internal iliac artery (Discovery IGS 740, GE Healthcare, Chicago, IL). Maximum Intensity Projection (MIP) coronal views showing the left pelvic arterial vascular anatomy (a) and the left prostatic artery (arrow) and prostate gland blush (b)

as much distal information of the prostatic arteries. Based on this experience, and given the lower prevalence of type V cases (de Assis et al. 2015), the recommendation is to start straightforwardly with the internal iliac artery CBCT. It provides a rich source of anatomical information with the adequate level of distality and selectivity in most cases. In practice, CBCTs can be acquired arms down or with arms on the chest with limited impact on image quality thanks to automatic exposure optimization (IGS5/740, GE Healthcare).

Proximal CBCT datasets should be analyzed carefully to identify arteries feeding the prostate, their pathways and non-target vessels. Prostatic arteries should be identified exhaustively and bilaterally to maximize treatment completion and reduce symptom recurrence risks (de Assis et al. 2019a). Advanced planning software such as Virtual Injection (Embo ASSIST, GE Healthcare) allows to simulate selective injections based on a proximal $\mathrm{CBCT}$, facilitating the identification of prostatic arteries and non-target vessels (Fig. 3). Automatic segmentation of the pelvic and prostatic vasculature and of the arteries of interest, both prostate feeders and non-target vessels, along with their centerlines, facilitates procedure planning from table side and creates a 3D model for augmented fluoroscopy (Fig. 3).

During microcatheter navigation $(\leq 2.4$ French recommended), this 3D model is overlaid on the live fluoroscopy, providing a 3D roadmap that is and remains automatically registered to the patient despite gantry and table movements. In case of patient motion, the 3D roadmap registration can be adjusted from table side to better match the patient. Depending on the procedure stage, both the prostatic artery to select and the nontarget arteries to avoid can be interesting to display on fluoroscopy. In addition to its benefits facilitating catheter guidance, the availability of the 3D roadmap allows to select the best working angulations to visualize vessel turns and bifurcations without using fluoroscopy thus with no radiation. The 45 degree ipsilateral angle typically recommended should be replaced by a systemic identification of the optimal working angle based on patient's anatomy using 3D roadmap without fluoroscopy, which may be less steep than 45 degrees and thus more radiation effective. Multiple DSA runs can thus be avoided by using the CBCT-based 3D roadmap, resulting in both dose and contrast savings.

During microcatheter navigation, basic ALARA radiation best practices should also be enforced to minimize radiation exposure both to patients and medical staff: use of digital zoom at maximum FOV instead of magnification; rigorous collimation; low default dose settings and frame rates, e.g. 1 fps for DSA and $3.75 \mathrm{fps}$ for fluoro, which can be increased when needed from table side; fluoroscopy storing instead of DSA when sufficient in terms of image quality; and avoiding steep angulations when possible to minimize scatter radiation and optimize image quality.

Once the microcatheter is in the desired location within the prostatic artery, a distal DSA in ipsilateral oblique view with $3-5 \mathrm{cc}$ of $50-70 \%$ contrast hand-injected or power-injected at 0.2 to $1 \mathrm{cc} / \mathrm{s}$ (depending on the prostate size, prostatic artery diameter and collaterals) is recommended to confirm no non-target vessels are being perfused, as well as to simulate the embolization treatment by visualizing the prostate perfusion. When further confirmation is needed, e.g. to clearly distinguish prostatic from rectal branches, or if both central gland and peripheral zone arterial branches are not observed, 


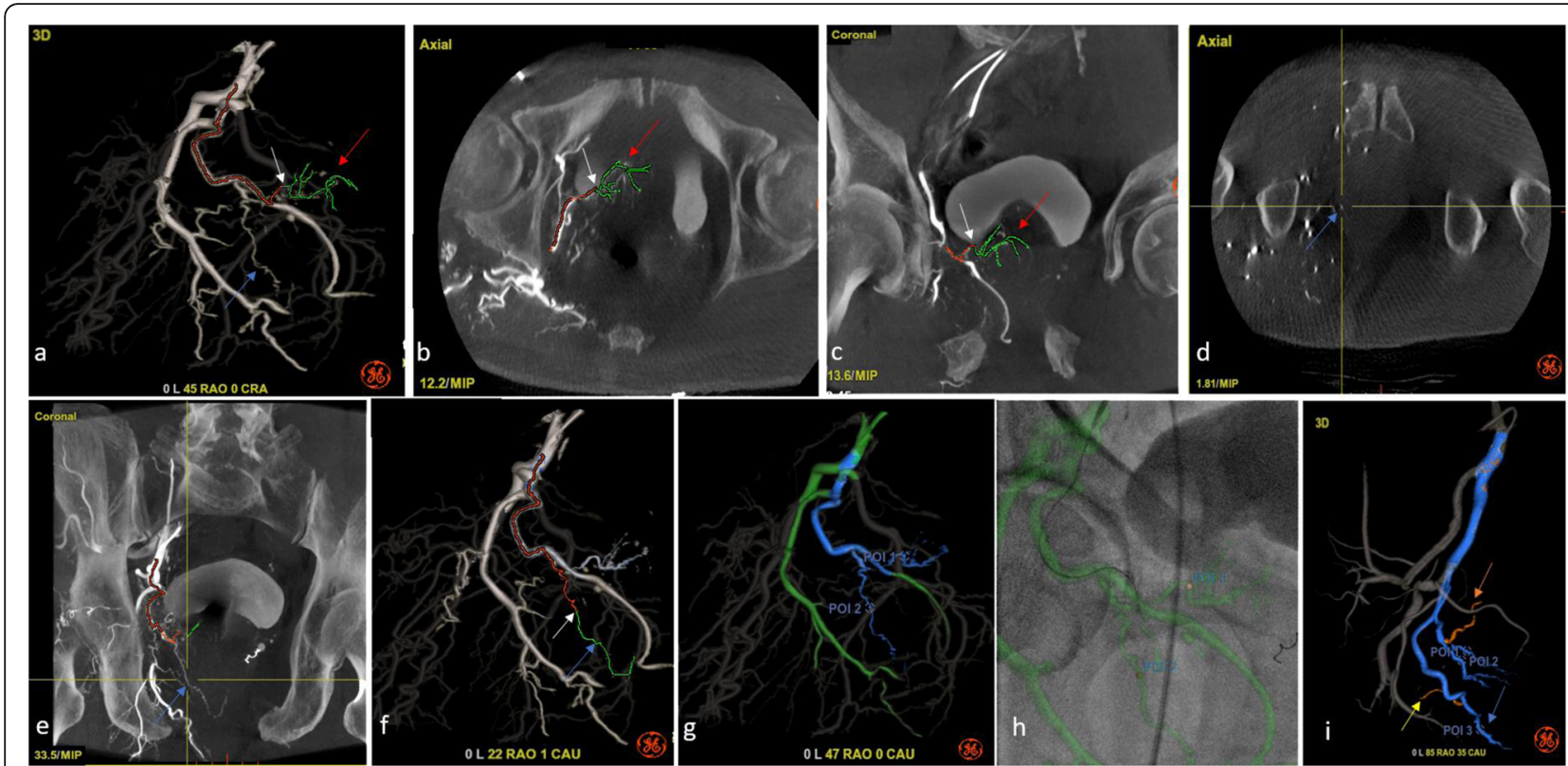

Fig. 3 Advanced planning on proximal CBCT using Virtual Injection technology (Embo ASSIST, GE Healthcare). Automatic arterial segmentation and bone removal, with Virtual Injection used to highlight the main prostatic artery (red arrow) (a-c). Distality from, and path to the virtual injection point (white arrow) are indicated in green and red, respectively. A separate secondary prostatic artery (blue arrow) was thus detected since unperfused by the injection simulation (d-f). This branch was initially taken to be a rectal branch on the 3D volume rendering $(\mathbf{a})$, but confirmed as a prostatic artery with Virtual Injection (f). Both prostatic arteries were saved as 3D guidance model ( $\mathbf{g}$ ) to facilitate their catheterization using augmented fluoroscopic guidance (h). Prostatic arteries are indicated in blue, with planned points of injection (POI) marked on the overall navigation 3D model (in green). Separate case (i) showing how advanced planning with Virtual Injection allowed detection of a bladder branch (orange arrow) arising from the central prostatic artery, inducing the need for two separate distal points of injection (POls 1 \& 2), as well as a capsular branch (blue arrow), with POI3 defined distally from a branch going to the rectum (yellow arrow). 3D model for augmented fluoroscopic guidance highlighting path to prostatic arteries (in blue), 3 planned points of embolization, and non-target branches to avoid (in orange)

or for early experience PAE users, a distal $5 \mathrm{~s}$ CBCT is recommended with either hand or power injection with an $8-10$ s delay to ensure good filling of both the prostate and any extra-prostatic structure (Fig. 4). In this group's experience, hand injection gives better control to fill the prostate, avoid reflux and obtain a strong injection to identify shunts. For hand injected CBCTs, half of the contrast is injected before the spin starts to optimize contrast uptake in the prostate and surrounding organs; the other half is injected during the spin rotation to confirm the local arterial anatomy and help evaluate risks of reflux in non target anatomies. Operators should not be present in the examination room during $\mathrm{CBCT}$ acquisition. If required due to manual injection preferrence, operators should stand behind lead shields to minimize their own radiation exposure. To avoid operator presence in examination room, power injection with PSI at 300 can help avoid reflux during CBCT acquisition.

Intra-arterial vasodilator should be used when navigating stenotic and/or tortuous anatomies, before DSA/ $\mathrm{CBCT}$ acquisition and before embolic agents injection, with the aim of opening the prostatic vascular bed. Isosorbide mononitrate (5-10 mg) and nitroglycerin $(100 \mu \mathrm{g})$ are typically used. Prostate enhancement can be

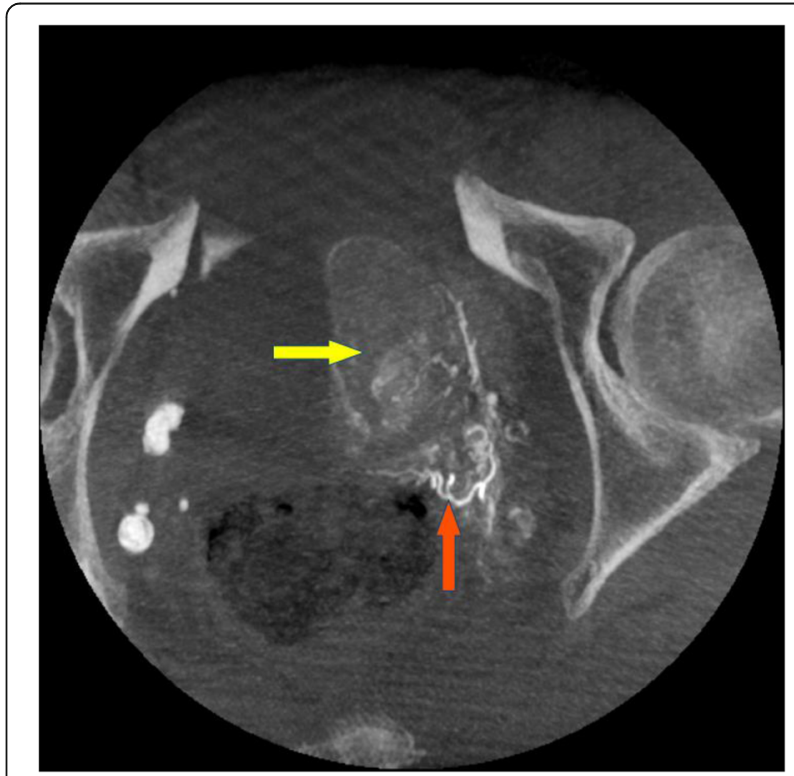

Fig. 4 Distal CBCT. Ten millimeters MIP axial view from a superselective CBCT showing prostatic blush (yellow arrow) as well as rectal branch (orange arrow) originating from a prostatic artery, observed after vasodilator injection. The catheter had to be repositioned to avoid off-target embolization 
better observed after vasodilator injection. Some shunts, mostly common to the internal pudendal artery, rectum and bladder, can also be more evident after vasodilator injection.

Once distal DSA/CBCT analysis confirms the microcatheter location for treatment, the embolic material is delivered proximally first, then deeper in the gland, following the PErFecTED technique (Carnevale et al. 2020; Zumstein et al. 2020). Postero-anterior incidence can be used for types I, II and III with the aim of reducing radiation exposure because they are longer and there is less risk of embolic agent reflux in these types of arteries. Ipsilateral oblique view is recommended for type IV because of the risk of proximal embolic agent reflux to the internal pudendal artery. Microspheres ranging from 100 to $500 \mu \mathrm{m}$ have been used. Adverse events and complications seem to be more frequent with smaller particles due to deeper penetration and passing through anastomosis (Carnevale et al. 2020; Gonçalves et al. 2016). During this time-consuming step of embolic agent injection, low dose fluoroscopy at $3.75 \mathrm{fps}$ can be used to monitor the embolization, in unsubtracted or subtracted mode, and stored for documentation purposes. Intermittent $0.5 \mathrm{fps}$ DSAs can be used as necessary. Collimation, digital zoom instead of magnification, and use of posteroanterior views are particularly recommended to reduce radiation in this step. A final 0.5fps DSA or stored fluoroscopy allows to control the success of the embolization, defined as total stasis. The catheter is then navigated to the other side where the workflow is repeated.

\section{Post-procedural imaging}

For post-PAE it's recommended pelvic US to measure the postvoid residual volume and prostate size. MRI should be used to a better understanding of the ischemic areas and prostate reduction, mainly in patients with poor results or for research's aims. The recommendation is 3 months after the procedure and then an annual follow up.

\section{Conclusion}

Prostatic artery embolization has demonstrated to deliver quality of life and symptoms improvements, reducing the size and consistency of the prostate (de Assis et al. 2019b) with limited side effects compared to surgery. However, little has been published on optimal imaging to guide this technically challenging procedure.

This technical note puts forth a consensus optimized imaging workflow and best practices, with the hope of helping drive adoption of the procedure, deliver good clinical outcomes, and minimize radiation dose levels and contrast media injections while making PAE procedures shorter and safer. This assumption will be addressed in future studies.

\section{Abbreviations}

TURP: Transurethral resection of the prostate; PAE: Prostatic artery embolization; BPH: Benign prostatic hyperplasia; CBCT: Cone-beam computed tomography; DSA: Digital subtraction angiography; LUTS: Lower urinary tract symptoms; CT: Computed tomography; MR: Magnetic resonance

\section{Acknowledgements}

The authors would like to thank Raphael Doustaly and Lisa Lewis for their clinical support, and Meryem Idrissi and Laurence Gavit for assistance in preparation of the manuscript.

Presented at SIR 2020 (poster 818).

\section{Authors' contributions}

FCC, TM, W, AMA, AMM, CN discussed and evaluated their best imaging and technical practices when performing prostatic artery embolization procedures and reached a consensus. FC, ADDR and AR intermediated all the discussions and were major contributors in writing the manuscript. All authors read and approved the final manuscript.

\section{Funding}

No source of funding.

Availability of data and materials

Not applicable.

\section{Declarations}

Ethics approval and consent to participate

Retrospective analysis approved by local institutional review boards, exempted from patient informed consent.

Consent for publication

Not applicable.

\section{Competing interests}

FCC, AMA, AMM, ADDR and TM receive a research grant from GE Healthcare. FCC, TM and CN received consulting fees from GE Healthcare outside of this work. AR is a GE Healthcare employee. The other authors have nothing to disclose.

\section{Author details}

'Department of Radiology, University of Sao Paulo Medical School, Av. Dr. Enéas Carvalho de Aguiar, 255, São Paulo, SP 05403-000, Brazil. Weill Cornell Medicine, New York, NY, USA. ${ }^{3}$ La Timone Hospital, Marseille, France. ${ }^{4} \mathrm{GE}$ Medical Systems, Buc, France. ${ }^{5}$ Endovascular Consultants of Colorado Lone Tree, Lone Tree, Colorado, USA.

Received: 11 May 2021 Accepted: 26 July 2021

Published online: 10 August 2021

\section{References}

Andrade G, Khoury HJ, Garzon WJ et al (2017) Radiation exposure of patients and interventional radiologists during prostatic artery embolization: a prospective single-operator study. J Vasc Interv Radiol 28(4):517-521. https://doi.org/10.1 016/j.jvir.2017.01.005

Bagla S, Rholl KS, Sterling KM, van Breda A, Papadouris D, Cooper JM, van Breda A (2013) Utility of cone-beam CT imaging in prostatic artery embolization. J Vasc Interv Radiol 24(11):1603-1607. https://doi.org/10.1016/j.jvir.2013.06.024

Bagla S, Smirniotopoulos J, Orlando J et al (2017) Cost analysis of prostate artery embolization (PAE) and transurethral resection of the prostate (TURP) in the treatment of benign prostatic hyperplasia. Cardiovasc Intervent Radiol 40: 1694-1697. https://doi.org/10.1007/s00270-017-1700-7.

Bagla S, Sterling KM (2014) Pitfalls of cone beam computed tomography in prostate artery embolization. Cardiovasc Intervent Radiol 37(6):1430-1435. https://doi.org/10.1007/s00270-014-0982-2

Bilhim T, Casal D, Furtado A, Pais D, O'Neill JE, Pisco JM et al (2011a) Branching patterns of the male internal iliac artery: imaging findings. Surg Radiol Anat 33(2):151-159. https://doi.org/10.1007/s00276-010-0716-3

Bilhim T, Pisco JM, Furtado A (2011b) Prostatic arterial supply: demonstration by multirow detector angio CT and catheter angiography. Eur Radiol 21(5): 1119-1126. https://doi.org/10.1007/s00330-010-2015-0 
Brown AD, Stella SF, Simons ME (2019 Apr) Minimally invasive treatment for benign prostatic hyperplasia: economic evaluation from a standardized hospital case costing system. Cardiovasc Intervent Radiol 42(4):520-527. https://doi.org/10.1007/s00270-018-2132-8

Cadour F, Tradi F, Habert P et al (2020) Prostatic artery embolization using threedimensional cone-beamcomputed tomography. Diagn Interv Imaging 101(11):721-725. ISSN 2211-5684, https://doi.org/10.1016/j.diii.2020.05.002.

Carnevale FC, Antunes AA (2013) Prostatic artery embolization for enlarged prostates due to benign prostatic hyperplasia. How I do it. Cardiovasc Intervent Radiol 36(6):1452-1463. https://doi.org/10.1007/s00270-013-0680-5

Carnevale FC, da Motta-Leal-Filho JM, Antunes AA, Baroni RH, Marcelino ASZ, Cerri LMO, Yoshinaga EM, Cerri GG, Srougi M (2013 Apr) Quality of life and clinical symptom improvement support prostatic artery embolization for patients with acute urinary retention caused by benign prostatic hyperplasia. J Vasc Interv Radiol 24(4):535-542. https://doi.org/10.1016/j.jvir.2012.12.019

Carnevale FC, Iscaife A, Yoshinaga EM, Moreira AM, Antunes AA, Srougi M (2016) Transurethral resection of the prostate (TURP) versus original and PErFecTED prostate artery embolization (PAE) due to benign prostatic hyperplasia (BPH): preliminary results of a single center, prospective, urodynamic controlled analysis. Cardiovasc Intervent Radiol 39(1):44-52. https://doi.org/10.1007/s00270-015-1202-4

Carnevale FC, Moreira AM, Antunes AA (2014) The "PErFecTED technique": proximal embolization first, then embolize distal for benign prostatic hyperplasia. Cardiovasc Intervent Radiol 37(6):1602-1605. https://doi.org/10.1 007/s00270-014-0908-z

Carnevale FC, Moreira AM, de Assis AM (2020) Prostatic artery embolization for the treatment of lower urinary tract symptoms due to benign prostatic hyperplasia: 10 years' experience. Radiology. 296(2):444-451. https://doi.org/1 0.1148/radiol.2020191249.

Carnevale FC, Soares GR, de Assis AM, Moreira AM, Harward SH, Cerri GG (2017) Anatomical variants in prostate artery embolization: a pictorial essay. Cardiovasc Intervent Radiol 40(9):1321-1337. https://doi.org/10.1007/s00270017-1687-0

Cornelis FH, Bilhim T, Hacking N, Sapoval M, Tapping CR, Carnevale FC (2020) CIRSE standards of practice on prostatic artery embolisation. Cardiovasc Intervent Radiol 43(2):176-185. https://doi.org/10.1007/s00270019-02379-3

de Assis AM, Maciel MS, Moreira AM, de Paula Rodrigues VC, Antunes AA, Srougi M, Cerri GG, Carnevale FC (2017) Prostate zonal volumetry as a predictor of clinical outcomes for prostate artery embolization. Cardiovasc Intervent Radiol 40(2):245-251. https://doi.org/10.1007/s00270-016-1518-8

de Assis AM, Moreira AM, Carnevale FC (2019a) Angiographic findings during repeat prostatic artery embolization. JVIR. 30(5):645-651. https://doi.org/10.1 016/j.jvir.2018.12.734

de Assis AM, Moreira AM, Carnevale FC, Marcelino ASZ, de Oliveira Cerri LM, Antunes AA, Srougi M, Cerri GG (2019b) Effects of prostatic artery embolization on the dynamic component of benign prostate hyperplasia as assessed by ultrasound elastography: a pilot series. Cardiovasc Intervent Radiol 42(7):1001-1007. https://doi.org/10.1007/s00270-019-02220-x

de Assis AM, Moreira AM, de Paula Rodrigues VC, Harward SH, Antunes AA, Srougi M, Carnevale FC (2015) Pelvic arterial anatomy relevant to prostatic artery embolization and proposal for angiographic classification. Cardiovasc Intervent Radiol 38(4):855-861. https://doi.org/10.1007/s00270-015-1114-3

Desai H, Yu H, Ohana E, Gunnell ET, Kim J, Isaacson A (2018) Comparative analysis of cone-beam CT angiogram and conventional CT angiogram for prostatic artery identification prior to embolization. J Vasc Interv Radiol 29(2): 229-232. https://doi.org/10.1016/j.jvir.2017.09.020 PMID: 29414195. 10.1016/j. jvir.2017.09.020

Gao YA, Huang Y, Zhang R, Yang YD, Zhang Q, Hou M, Wang Y (2014) Benign prostatic hyperplasia: prostatic arterial embolization versus transurethral resection of the prostate- - prospective, randomized, and controlled clinical trial. Radiology 270(3):920-928. https://doi.org/10.1148/radiol.13122803

Garzon WJ, Andrade G, Dubourcq F et al (2016) Prostatic artery embolization: radiation exposure to patients and staff. J Radiol Prot 36(2):246-254. https:// doi.org/10.1088/0952-4746/36/2/246

Gonçalves OM, Carnevale FC, Moreira AM, Antunes AA, Rodrigues VC, Srougi M (2016) Comparative study using 100-300 versus 300-500 $\mu \mathrm{m}$ microspheres for symptomatic patients due to enlarged-BPH prostates. Cardiovasc Intervent Radiol 39(10):1372-1378. https://doi.org/10.1007/s00270-016-1443-x

Guneyli S, Ward E, Thomas S, Yousuf AN, Trilisky I, Peng Y, Antic T, Oto A (2016) Magnetic resonance imaging of benign prostatic hyperplasia. Diagn Interv Radiol 22(3):215-219. https://doi.org/10.5152/dir.2015.15361
Hacking N, Vigneswaran G, Maclean D, Modi S, Dyer J, Harris M, Bryant T (2019) Technical and imaging outcomes from the UK registry of prostate artery embolization (UK-ROPE) study: focusing on predictors of clinical success. Cardiovasc Intervent Radiol 42(5):666-676. https://doi.org/10.1007/s00270-01 8-02156-8 Epub 2019 Jan 2. PMID: 30603967

Hertault A, Rhee R, Antoniou GA, Adam D, Tonda H, Rousseau H, Bianchini A, Haulon S (2018) Radiation dose reduction during EVAR: results from a prospective multicentre study (the REVAR study). Eur J Vasc Endovasc Surg 56(3):426-433. https://doi.org/10.1016/j.ejvs.2018.05.001

Kim AY, Field DH, DeMulder D et al (2018) Utility of MR angiography in the identification of prostatic artery origin prior to prostatic artery embolization. J Vasc Interv Radiol 29(3):307-310.e1

Laborda A, De Assis AM, loakeim I et al (2015) Radiodermitis after prostatic artery embolization: case report and review of the literature. Cardiovasc Intervent Radiol 38(3):755-759. https://doi.org/10.1007/s00270-015-1083-6

Lecumberri N, Gorbea I, de Ocariz GS et al (2018) Prostatic artery embolization versus transurethral resection of the prostate in the treatment of benign prostatic hyperplasia: protocol for a non-inferiority clinical trial. Res Rep Urol 10:17-22

Maclean D, Maher B, Harris M, Dyer J, Modi S, Hacking N, Bryant T (2018 Apr) Planning prostate artery embolisation: is it essential to perform a preprocedural CTA? Cardiovasc Intervent Radiol 41(4):628-632. https://doi.org/1 0.1007/s00270-017-1842-7

Maclean D, Maher B, Modi S, Harris M, Dyer J, Somani B, Hacking N, Bryant T (2017) Prostate artery embolization: a new, minimally invasive treatment for lower urinary tract symptoms secondary to prostate enlargement. Ther Adv Urol 9(8):209-216. https://doi.org/10.1177/1756287217717889

Rassweiler J, Teber D, Kuntz R, Hofmann R (2006) Complications of transurethral resection of the prostate (TURP)-incidence, management, and prevention. Eur Urol 50(5):969-979; discussion 980. https://doi.org/10.1016/j.eururo.2 005.12 .042

Ray A, Powell J, Speakman M et al (2018) Efficacy and safety of prostate artery embolization for benign prostatic hyperplasia: an observational study and propensity-matched comparison with transurethral resection of the prostate (the UK-ROPE study). BJU Int 122:270-282

Rocha A, de Assis AM, Moreira AM, Carnevale FC. Advantages of using conebeam computed tomography over digital subtraction angiography to identify prostatic arteries in prostatic artery embolization. SIR 2020. URL: https://www.sirmeeting.org/index.cfm?do=abs.viewAbs\&abs=1888. Accessed 2 Jul 2020.

Schnapauff D, Maxeiner A, Wieners G, Denecke T, Hamm B, Gebauer B, Jonczyk M (2020) Semi-automatic prostatic artery detection using cone-beam CT during prostatic arterial embolization. Acta Radiol 61(8):1116-1124. https:// doi.org/10.1177/0284185119891689 Epub 2019 Dec 12. PMID: 31830430

Schott P, Katoh M, Fischer N, Freyhardt P (2019) Radiation dose in prostatic artery embolization using cone-beam CT and 3D roadmap software. J Vasc Interv Radiol 30(9):1452-1458. https://doi.org/10.1016/j.jvir.2019.04.040 Epub 2019 Jul 29. PMID: 31371137

Tanaka M, Lacayo E, Katrivesis J, Spies J, Kim A (2017) Radiation doses in prostatic artery embolization for benign prostatic hypertrophy: a single-institution series and meta-analysis. J Vasc Interv Radiol 28(2):S149. https://doi.org/10.1 016/j.jvir.2016.12.962

Wang MQ, Duan F, Yuan K, Zhang GD, Yan J, Wang Y (2017 Jan) Benign prostatic hyperplasia: cone-beam CT in conjunction with DSA for identifying prostatic arterial anatomy. Radiology 282(1):271-280. https://doi.org/10.1148/radiol.201 6152415

Zumstein V, Binder J, Güsewell S, Betschart P, Pratsinis M, Müllhaupt G, Hechelhammer L, Schmid HP, Abt D (2020) Radiation exposure during prostatic artery embolisation: a systematic review and calculation of associated risks. Eur Urol Focus 7(3):608-611. https://doi.org/10.1016/j.euf.202 0.04 .012

\section{Publisher's Note}

Springer Nature remains neutral with regard to jurisdictional claims in published maps and institutional affiliations. 